\title{
Application of orthogonal array technique and particle swarm optimization approach in surface roughness modification when face milling AISI1045 steel parts
}

\author{
Masoud Azadi Moghaddam ${ }^{1}$ Farhad Kolahan ${ }^{1}$
}

Received: 23 March 2015/Accepted: 11 December 2015/Published online: 30 December 2015

(C) The Author(s) 2015. This article is published with open access at Springerlink.com

\begin{abstract}
Face milling is an important and common machining operation because of its versatility and capability to produce various surfaces. Face milling is a machining process of removing material by the relative motion between a work piece and rotating cutter with multiple cutting edges. It is an interrupted cutting operation in which the teeth of the milling cutter enter and exit the work piece during each revolution. This paper is concerned with the experimental and numerical study of face milling of AISI1045. The proposed approach is based on statistical analysis on the experimental data gathered using Taguchi design matrix. Surface roughness is the most important performance characteristics of the face milling process. In this study the effect of input face milling process parameters on surface roughness of AISI1045 steel milled parts have been studied. The input parameters are cutting speed $(v)$, feed rate $\left(f_{z}\right)$ and depth of cut $\left(a_{p}\right)$. The experimental data are gathered using Taguchi $\mathrm{L}_{9}$ design matrix. In order to establish the relations between the input and the output parameters, various regression functions have been fitted on the data based on output characteristics. The significance of the process parameters on the quality characteristics of the process was also evaluated quantitatively using the analysis of variance method. Then, statistical analysis and validation experiments have been carried out to compare and select the best and most fitted models. In the last section of this research, mathematical model has been developed for surface roughness prediction using particle
\end{abstract}

Masoud Azadi Moghaddam masoudazadi888@gmail.com; masoud_azadi88@yahoo.com

1 Department of Mechanical Engineering, Ferdowsi University of Mashhad, P.O. Box 91775-111, Mashhad, Iran swarm optimization (PSO) on the basis of experimental results. The model developed for optimization has been validated by confirmation experiments. It has been found that the predicted roughness using PSO is in good agreement with the actual surface roughness.

Keywords Face milling process - Surface roughness · Optimization · Particle swarm optimization (PSO) . Analysis of variance (ANOVA) - Taguchi approach

\section{Introduction}

The surface quality is one of the most specified customer requirements and the major indicator of surface quality on machined parts is surface roughness. The surface roughness is mainly a result of various controllable or uncontrollable process parameters and it is harder to attain and track than physical dimensions are. A considerable number of studies have researched the effects of the cutting speed, feed, depth of cut, and other factors on the surface roughness. In recent studies the effects of some factors on surface roughness has been evaluated and models has been developed. A central task in science and engineering practice is to develop models that give a satisfactory description of physical systems being observed (Baji et al. 2008; Azadi Moghaddam and Kolahan 2014; Bharathi Raja and Baskar 2012). The goal of this study is to obtain a mathematical model that relates the surface roughness to three cutting parameters in face milling, precisely to the cutting speed, feed rate and depth of cut (Baji et al. 2008).

There is various simple surface roughness amplitude parameters used in industry, such as roughness average $(\mathrm{Ra})$, root-mean-square (RMS) roughness $(\mathrm{Rq})$, and maximum peak-to-valley roughness (Ry or Rmax), etc. (Azadi 
Moghaddam and Kolahan 2014). The parameter Ra is used in this study. The average roughness $(\mathrm{Ra})$ is the area between the roughness profile and its mean line, or the integral of the absolute value of the roughness profile height over the evaluation. Therefore, the Ra is specified by the following equation (Azadi Moghaddam and Kolahan 2014):

$\mathrm{Ra}=\frac{1}{L} \int_{0}^{L}|Y(x)| \mathrm{dx}$

where $\mathrm{Ra}$ is the arithmetic average deviation from the mean line, $L$ the sampling length, and $Y$ is the ordinate of the profile curve (Fig. 1). There are many methods of measuring surface roughness, such as image processing, microscopes, stylus type instruments, profile tracing instruments, etc. (Azadi Moghaddam and Kolahan 2014).

There are a great number of factors influencing the surface roughness and Fig. 2 shows all influential factors on machined surface roughness (Baji et al. 2008).

Selection of appropriate machining parameters is an important step in the process planning of any machining operation. The present method of selection of machining parameters mainly depends either on previous work experience of the process planner or thumb rule or any machining data hand book. But it is a known fact that the machining parameters obtained from these resources are far from the optimal parameters and may be very much useful for theoretical investigations. The other possibility of selecting machining parameters is by conducting 'trial and error' experiments. But this act of experiments is purely non-technical and, moreover, time and cost are unnecessarily wasted for this purpose. The surface roughness of any manufacturing process has become critical because of increased quality demands. Sometimes, even if the dimensions of the component are well within the dimensional tolerances, still there are possibilities of

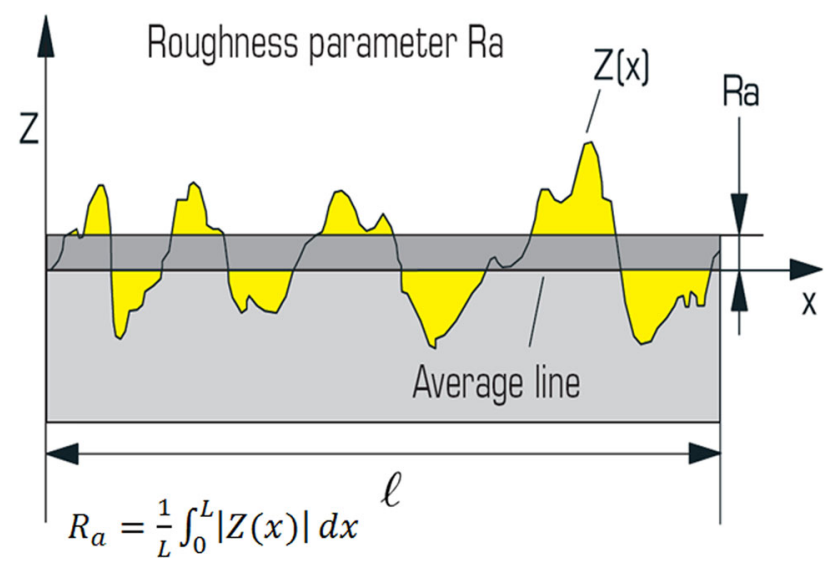

Fig. 1 The method used for calculation of SR (Azadi Moghaddam and Kolahan 2014) rejecting the component for the lack of required surface finish. Moreover, surface roughness determines mechanical properties such as wear, corrosion, lubrication, electrical conductivity and fatigue behavior. Surface roughness is an important measure of the quality of a product and also greatly influences the production cost. Production of required surface finish on a component is mainly dependent on many parameters such as cutting speed, feed, depth of cut, tool nomenclature, cutting force, rigidity of the machine and so on. Among these parameters, cutting speed, feed and depth of cut are parameters which are easily controllable during the process of machining (Bharathi Raja and Baskar 2012).

The main objectives of the present study are: (1) to establish the relationship between face milling process parameters and the process output characteristic (surface roughness), and (2) to determine the optimal parameter levels for minimum surface roughness by application of particle swarm optimization (PSO) algorithm. The proposed procedure is based on statistical analysis of the experimental data. The article concludes with the verification of the proposed approach and a summary of the major findings.

\section{Experimental details}

Review of the research work reveals that much work has been done on surface roughness of face milling process. These studies have mostly emphasized on the modeling and optimization of the process parameters (Jeang 2011; Patricia Mũnoz-Escalona and Maropoulos 2014; Reddy and Venkateswara 2006; Jesuthanam et al. 2007; Zhang et al. 2007; Bharathi Raja and Baskar 2012; Arrazola et al. 2013).

Jeang (2011) determined the optimal cutting parameters required to minimize the cutting time while maintaining an acceptable quality level. The equation for predicting cutting time was determined by CATIA software along with response surface methodology. The proposed approach could produce automatic product and process design that may lead to cost reduction and quality improvement.

Patricia Mũnoz-Escalona and Maropoulos (2014) reported a research study on the development of a geometrical model for surface roughness prediction when face milling with square inserts. The model is based on a geometrical analysis of the recreation of the tool trail left on the machined surface. The model has been validated with experimental data obtained for high speed milling of aluminum alloy (Al 7075-T7351) when using a wide range of cutting speed, feed per tooth, axial depth of cut and different values of tool nose radius $(0.8$ and $2.5 \mathrm{~mm})$, using the Taguchi method as the design of experiments. The 
Fig. 2 Fishbone diagram with factors that influence on surface roughness (Baji et al. 2008)

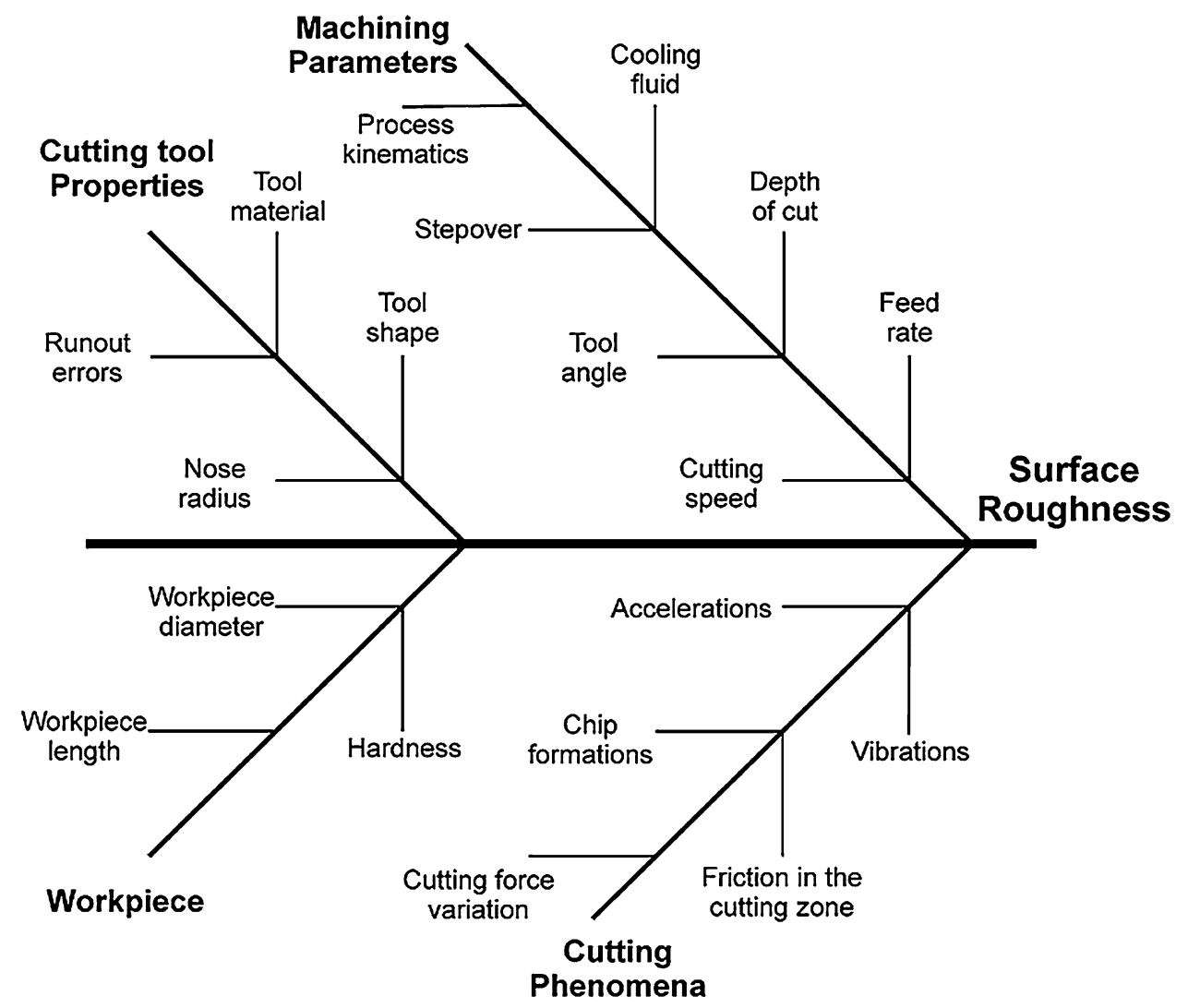

experimental roughness was obtained by measuring the surface roughness of the milled surfaces with a non-contact profile meter. The developed model can be used for any combination of material work piece and tool, when tool flank wear is not considered and is suitable for using any tool diameter with any number of teeth and tool nose radius. The results showed that the developed model achieved an excellent performance with almost $98 \%$ accuracy in terms of predicting the surface roughness when compared to the experimental data.

Reddy and Venkateswara (2006) studied the effect of tool geometry (radial rake angle and tool nose radius) and cutting conditions (cut-ting speed and feed rate) on the machining performance during end milling of medium carbon steel. First and second order mathematical models, in terms of machining parameters were developed for surface roughness prediction using RSM. The results showed that the cutting speed, the feed, the radial rake angle and the tool nose radius are the primary factors influencing the surface roughness of medium carbon steel during end milling processes.

Jesuthanam et al. (2007) proposed the development of a novel hybrid neural network (NN) trained with genetic algorithm (GA) and particle swarm optimization (PSO) for the prediction of surface roughness. The proposed hybrid NN was found to be competent in terms of computational speed and efficiency over the NN model.
Zhang et al. (2007) studied the Taguchi design application to optimize the surface quality of a face milling operation when using a CNC. The results verified that the Taguchi design was successfully in optimizing the milling parameters for surface roughness.

Bharathi Raja and Baskar (2012) developed a generalized model based on particle swarm optimization (PSO) technique to achieve a desired surface roughness when face milling aluminum. The machining time was included as input parameter together with cutting speed, feed and depth of cut. They concluded that the use of optimization technique replaces the selection of cutting parameters by trial and error method.

Finally, Arrazola et al. (2013) compiled different advances in the modeling of machining processes. In its paper the advances in predictive, analytical, computational and empirical models among others for the prediction of variables such as surface roughness, cutting forces, stresses, chip formation, etc. are highlighted.

From analyzing all the literature, it has been observed that the proposed models are based on computational, numerical analysis and complex mathematical calculus. Based on these findings, the aim of this research is:

1. To develop a model for surface roughness prediction based on experiments gathered Taguchi technique, where also parameters such as cutting speed $(v)$, feed rate $\left(f_{z}\right)$ and depth of cut $\left(a_{p}\right)$ are considered. 
2. To derive the optimal parameter levels for minimum SR using statistical analysis of the experimental data and partial swarm optimization technique. Finally, the article concludes with the verification of the proposed approach and a summary of the major findings.

\section{Conditions of experiment}

Test samples made of AISI1045 steel parts with dimensions $15 \times 60 \times 80 \mathrm{~mm}$ were prepared and used in the experiments. The face milling experiments were performed

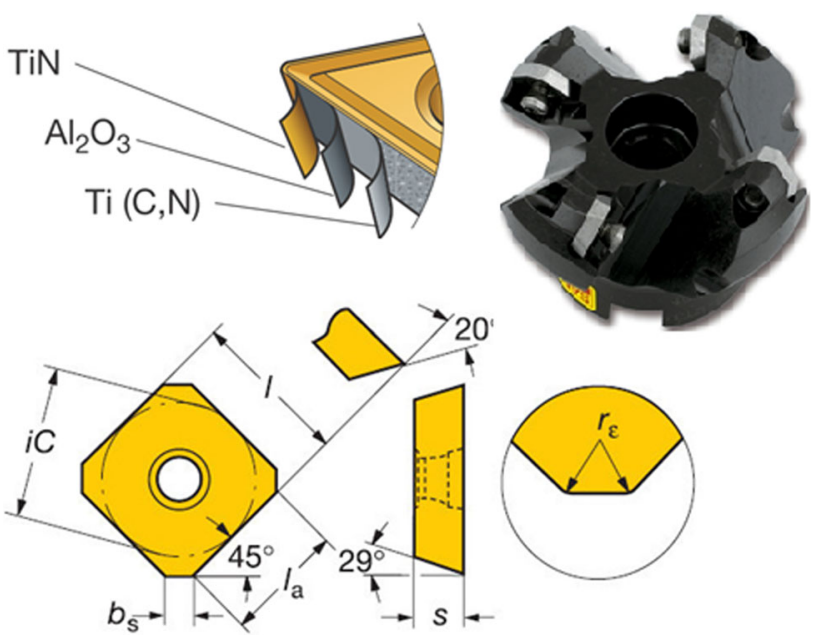

Fig. 3 Cutting tool used for machining

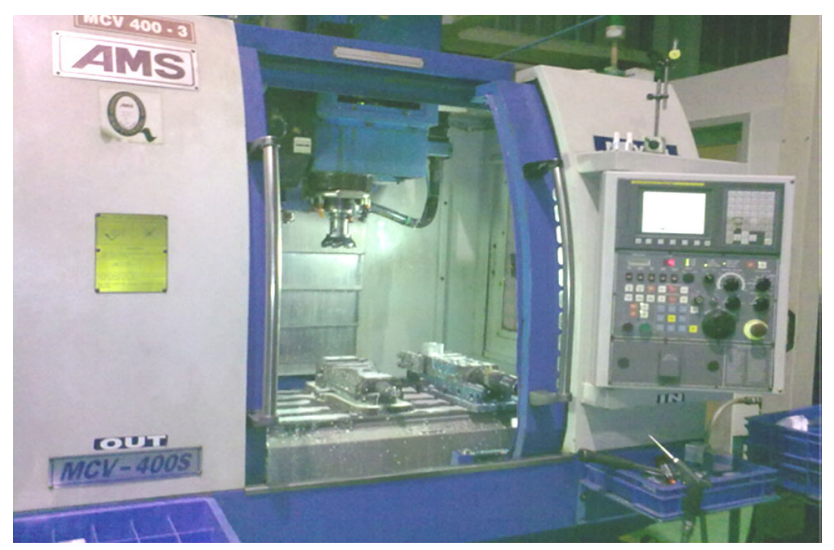

Fig. 4 CNC milling machine used by a tool for the face milling R245-12 T3M-PM 4020 using inserts with four helical right-hand cutting edges, produced by Iscar (Fig. 3). The experiments were conducted on MCV 400 CNC milling machine (Fig. 4). The feasible range for cutting parameters is taken from the machine limitations. The surface roughness tester is used to measure the roughness of the milled work piece. The measured surface roughness was obtained by averaging the surface roughness values at a minimum of three locations on the milled surface. A cut-off value of $8 \mathrm{~mm}$ was used when measuring the surface roughness of the milled surface.

\section{Process parameter setting}

A challenging task in any process is the selection of optimum machining parameter combinations for obtaining higher accuracy due to process variables and complicated process mechanisms.

In design of experiments (DOE), the number of required experiments (and hence the experiment cost) increases as the number of parameters and/or their corresponding levels increase. That is why it is recommended that the parameters with less likely pronounced effects on the process outputs be evaluated at fewer levels. In addition, the limitations of test equipment may also dictate a certain number of levels for some of the process parameters. For this research a lot of experiments had been done to find the relatively appropriate machine tool parameters and their proper settings as shown in Table 1.

\section{Taguchi technique}

Taguchi technique constructed a special set of general designs for factorial experiments that overcomes the drawbacks of partial factorial experiment. The method is popularly known as Taguchi's method. The special set of designs consists of orthogonal arrays (OA). The OA is a method of setting up experiments that only require a fraction of full factorial combinations. The treatment combinations are chosen to provide sufficient information to determine the factor effects using the analysis of means. Orthogonal refers to the balance of the various combinations of factors so that no one factor is given more or less weight in the experiment than the other factors. Orthogonal also refers to the fact that effect of each factor can be mathematically assessed independent of the effect of the
Table 1 Machining parameters and levels

\begin{tabular}{lllclll}
\hline Parameters & Symbol & Unit & Range & Level 1 & Level 2 & Level 3 \\
\hline Cutting speed $(v)$ & $C$ & $\mathrm{~m} / \mathrm{min}$ & $126-314$ & 126 & 201 & 314 \\
Feed rate $\left(f_{z}\right)$ & $F$ & $(\mathrm{~mm} / \mathrm{rev} \times$ tooth $)$ & $0.06-0.18$ & 0.06 & 0.12 & 0.18 \\
Depth of cut $\left(a_{p}\right)$ & $D$ & $(\mathrm{~mm})$ & $1-2$ & 1 & 1.5 & 2 \\
\hline
\end{tabular}


Table 2 The process characteristics an their corresponding output after 31 machining pass

\begin{tabular}{lllll}
\hline No & $V(\mathrm{~m} / \mathrm{min})$ & $f_{z}(\mathrm{~mm} /(\mathrm{rev} \times$ tooth $))$ & $a_{p}(\mathrm{~mm})$ & $\mathrm{SR}(\mu \mathrm{m})$ \\
\hline 1 & 1 & 1 & 1 & 1.67 \\
2 & 1 & 2 & 2 & 2.14 \\
3 & 1 & 3 & 3 & 2.22 \\
4 & 2 & 1 & 2 & 1.47 \\
5 & 2 & 2 & 3 & 2.04 \\
6 & 2 & 3 & 1 & 1.71 \\
7 & 3 & 1 & 3 & 1.75 \\
8 & 3 & 2 & 1 & 1.50 \\
9 & 3 & 3 & 2 & 1.94 \\
\hline
\end{tabular}

other factors. Taguchi's method, firstly, clearly defines orthogonal arrays, each of which can be used for many experimental situations. Secondly, Taguchi's method provides a standard method for analysis of results. Taguchi's method provides consistency and reproducibility that is generally not found in other statistical methods (Roy 2010).

This study has been undertaken to investigate the effects of cutting speed $(v)$, feed rate $\left(f_{z}\right)$ and depth of cut $\left(a_{p}\right)$ on surface roughness (SR). Therefore, $\mathrm{L}_{9}\left(3^{3}\right)$ design matrix has been used to carry out experiments (Table 2). Three process (input) parameters have been selected on the basis of literature survey and preliminary investigations. Preliminary experiments were conducted for the wide range of cutting speed, feed rate and depth of cut. Satisfactory results were obtained for $126-314 \mathrm{~m} / \mathrm{min}$, the range of cutting speed. Similar observations were made for specified range of feed rate and depth of cut.

Table 2 lists the machining parameters, their levels and their corresponding output.

\section{Mathematical modeling}

Regression models can be used to predict the behavior of input variables (independent variables) and values associated with each test response results (Kolahan et al. 2012).

The last column of Table 2 is the output for each test setting. These data can be used to develop mathematical models. Any of the above output is a function of process parameters which are expressed by linear, curvilinear or logarithmic functions; as stated in Eqs. 2-4, respectively.

$$
\begin{aligned}
Y_{1}= & b_{0}+b_{1} C+b_{2} F+b_{3} D \\
Y_{2}= & b_{0}+b_{1} C+b_{2} F+b_{3} D+b_{11} C C+b_{22} F F \\
& +b_{33} D D+b_{12} C F+b_{13} C D+b_{23} F D \\
Y_{3}= & b_{0} \times C^{b 1} \times F^{b 2} \times D^{b 3}
\end{aligned}
$$

In the above formula $b_{0}, b_{1}, b_{2}$ and $b_{3}$ are the regression coefficients to be estimated. In this study, based on the SR data given in Table 2, the regression model is developed using MINITAB software.

The choice of the model depends on the nature of initial data and the required accuracy. Using regression technique, in MINITAB Software, three types of mathematical functions (linear, curvilinear and logarithmic) have been fitted to the experimental data (Asokan et al. 2008): Models representing the relationship between process parameters and output characteristics can be stated in Eqs. 5-7.

Stepwise elimination process was used to modify the initial proposed models.

\section{Linear model}

$\mathrm{SR}(\mathrm{Ra})=1.23-0.00138 \times v+2.72 \times f_{z}+0.377 \times a_{p}$

\section{Curvilinear model}

$$
\begin{aligned}
\mathrm{SR}(\mathrm{Ra})= & 2.02-0.0125 \times V+13.5 \times f_{z}+0.000019 \\
& \times V^{2}-42.2 \times f_{z}^{2}+0.00184 \times V \times a_{p}
\end{aligned}
$$

\section{Logarithmic model}

$\mathrm{SR}(\mathrm{Ra})=5.585 \times V^{-0.163} \times f_{z}^{0.168} \times a_{p}^{0.296}$

Adequacies of models were checked by analysis of variance (ANOVA) technique within the confidence limit of $95 \%$ (Vishwakarma and Vishal Parashar 2012; Vishnu et al. 2013; Kolahan and Bironro 2008). Results are shown in Table 3. Given the required confidence limit $(\operatorname{Pr})$, the correlation factor $\left(R^{2}\right)$ and the adjusted correlation factor $\left(R^{2}\right.$-adj) for these models, it is evidence that Curvilinear model is superior to other two, thus, this model is considered as the best representative of the authentic milling process throughout in this paper.

In the next step, the proposed models were validated using new set of experiments (Table 4). Table 5 illustrates the mean error of the new six experiments for the output characteristics. According to the results the curvilinear model is the best model among the proposed models for surface roughness.

For illustrative purposes, the distribution of real data around regression lines for curvilinear model is illustrated

Table 3 ANOVA results for SR

\begin{tabular}{llllll}
\hline Model & Variable & $R^{2}(\%)$ & $R^{2}(\operatorname{adj})(\%)$ & $F$ value & $\operatorname{Pr}>F$ \\
\hline Linear & SR & 80.6 & 68.9 & 40.81 & $<0.0001$ \\
Curvilinear & SR & 98.4 & 95.1 & 79.46 & $<0.0001$ \\
Logarithmic & SR & 75.9 & 73.6 & 59.68 & $<0.0001$ \\
\hline
\end{tabular}


Table 4 New process variables for model validation

\begin{tabular}{llll}
\hline NO & $v$ & $f_{z}$ & $a_{p}$ \\
\hline 1 & 250 & 0.08 & 1.4 \\
2 & 250 & 0.10 & 1.6 \\
3 & 250 & 0.08 & 1.8 \\
4 & 250 & 0.10 & 1.8 \\
5 & 250 & 0.08 & 1.6 \\
6 & 250 & 0.10 & 1.4 \\
\hline
\end{tabular}

Table 5 Results of validation experiments

\begin{tabular}{llll}
\hline Machining parameters & \multicolumn{3}{l}{ Error $(\%)$} \\
\cline { 2 - 4 } & Linear & Logarithmic & Curvilinear \\
\hline SR & 6.97 & 8.82 & 3.54 \\
\hline
\end{tabular}

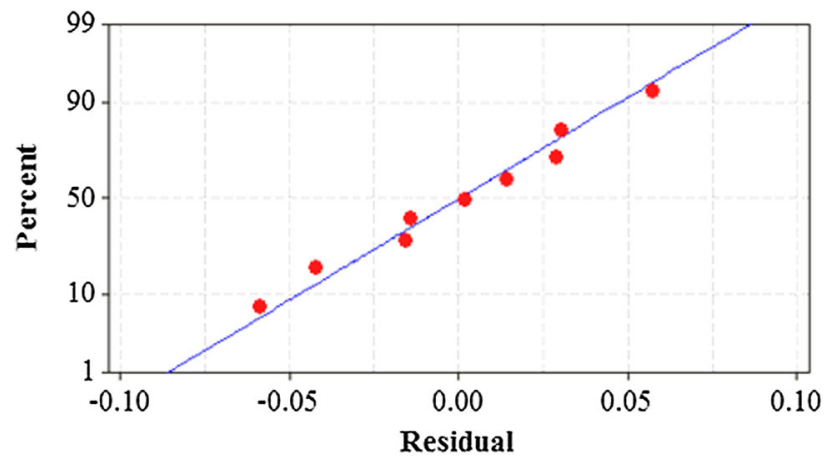

Fig. 5 Curvilinear model: actual values versus predicted values of SR

in Fig. 5. This figure demonstrates a good conformability of the developed model to the real process.

Figure 6 illustrates the predicted versus actual surface roughness for the model used.

Figure 7 demonstrates the interaction effect of cutting speed and feed rate (depth of cut remained constant). As illustrated, by increasing the feed rate within the range of $0.06-0.18 \mathrm{~mm} / \mathrm{rev} \times$ tooth, the SR increases. Similarly by increasing the cutting speed, within the range of $126-314 \mathrm{~m} / \mathrm{min}$, the SR decreases.

Figure 8 demonstrates the interaction effect of cutting speed and depth of cut on SR. As illustrated, by increasing the depth of cut within the range of 1-2 $\mathrm{mm}$, the SR increases, Similarly by increasing the cutting speed, within the range of $126-314 \mathrm{~m} / \mathrm{min}$, the SR decreases.

Figure 9 demonstrates the interaction effect of depth of cut and feed rate on SR. As illustrated, by increasing the depth of cut within the range of 1-2 $\mathrm{mm}$, the SR increases, Similarly by increasing the feed rate, within the range of $0.06-0.18 \mathrm{~m} / \mathrm{min}$, the SR increases.

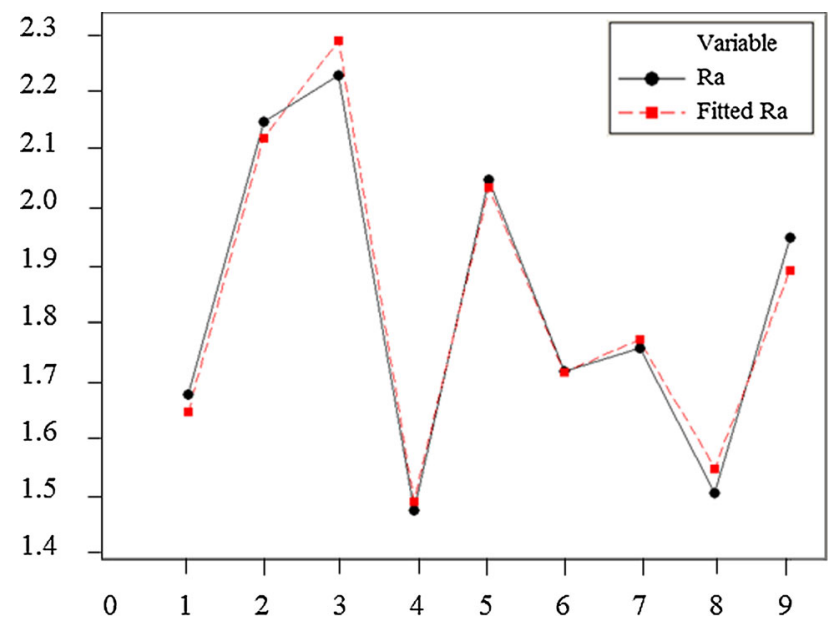

Fig. 6 Predicted versus actual surface roughness

\section{Analysis of variance (ANOVA)}

The ANOVA is used to investigate the most influential parameters to the process factor-level response. In this investigation, the experimental data are analyzed using the contribution rate. ANOVA has been performed on the above model to assess their adequacy, within the confidence limit of $95 \%$. ANOVA results indicate that the model is adequate within the specified confidence limit. The calculated determination coefficient $\left(R^{2}\right)$ for this model is $98.1 \%$. The result of ANOVA is shown in Table 3.

According to ANOVA procedure, large contribution rate indicates that the variation of the process parameter makes a big change on the performance characteristics (Table 6). In this study, a confidence level of $95 \%$ is selected to evaluate parameters significances (Azadi Moghaddam and Kolahan 2014).

ANOVA results may provide the percent contributions of each parameter (Roy 2010).

$P_{i}(\%)=\frac{\mathrm{SS}_{i}-\left(\mathrm{DOF}_{i} \times \mathrm{MS}_{\text {error }}\right)}{\text { Total Sum of Square }}$

In the above formula according to the ANOVA results (Table 6), $P_{i}$ is contribution percentage, $\mathrm{SS}_{i}$ is sum of square, $\mathrm{DOF}_{i}$ is degree of freedom of ith factor, and $\mathrm{MS}_{\text {error }}$ is mean sum of square of error (Azadi Moghaddam and Kolahan 2014).

The percent contributions of the milling parameters on SR is shown in Fig. 10. According to Fig. $10, v \times a_{p}$ is the major factor affecting the SR with $41 \%$ contribution. It is followed by $f_{z}, v, v \times v, f_{z} \times f_{z}$ with $29,24,1$ and $1 \%$ respectively. The remaining ( $4 \%$ ) effects are due to noise factors or uncontrollable parameters. 
Fig. 7 interaction of $v$ and $f_{z}$ plot for SR

Fig. 8 interaction of $v$ and $a_{p}$ plot for SR

\section{Proposed methodology}

The machining parameters are identified and the best combinations of parameters are to be selected by the optimization technique. Different optimization techniques have been used so far to solve the mathematical models for machining problems. Based on the previous literatures, the PSO technique always yielded best result as compared to other techniques. So the PSO technique is proposed to solve the mathematical model in this work.

\section{Particle swarm optimization}

Particle swarm optimization is a population-based stochastic optimization technique developed by Dr. Eberhart and Dr. Kennedy in 1995, inspired by social behavior of birds flocking (Bharathi Raja and Baskar 2012). The
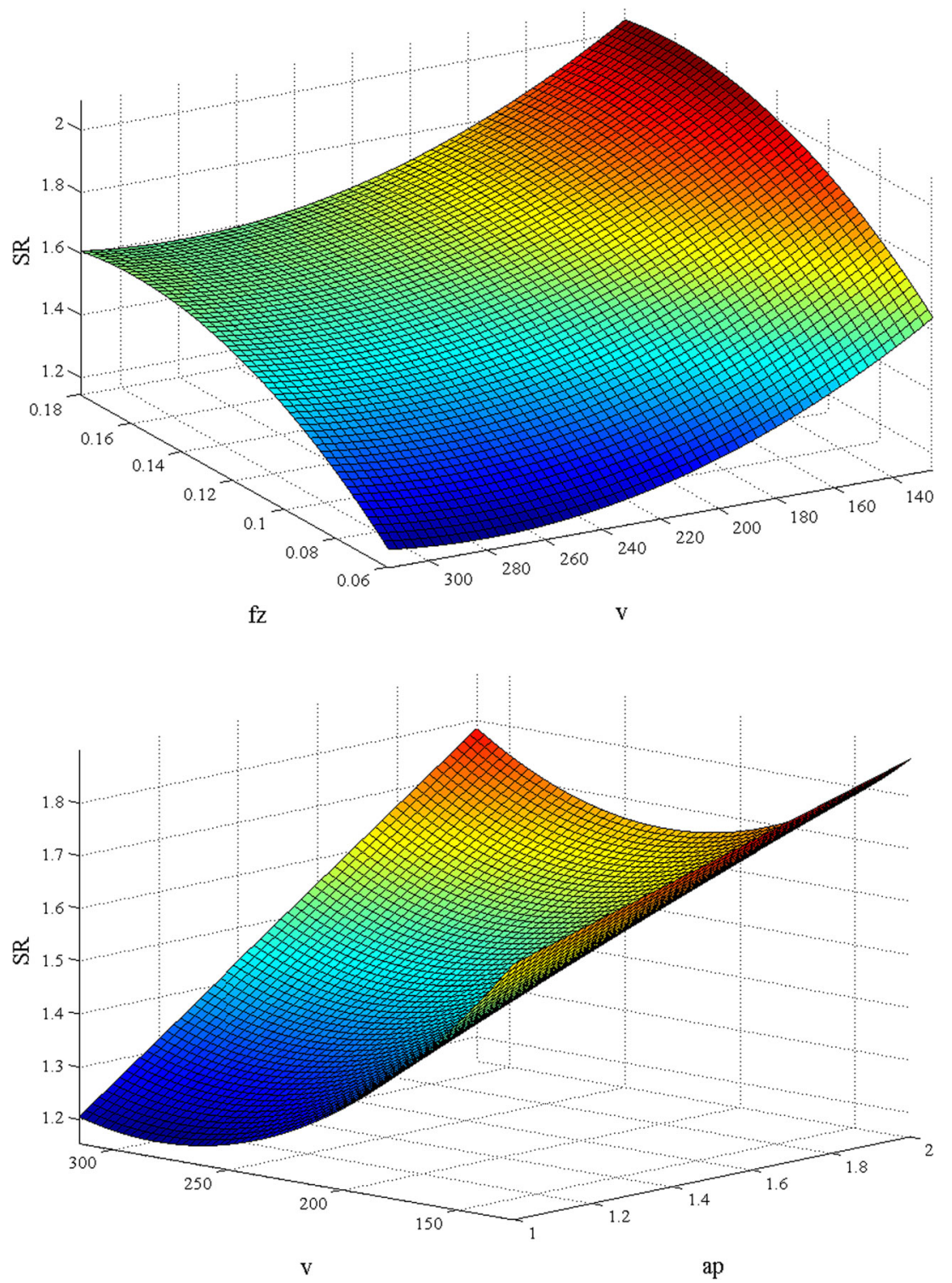

V

intelligence of swarm is based on the principle of social and psychological behavior of the swarm. The optimization procedure is initialized with a population of random solutions and searches for optima by updating generations. The potential solutions called particles fly through the problem space by following the current optimum particles. PSO is very easy to implement and there are few parameters to adjust. The algorithm can be explained based on the following scenario: a group of birds are randomly searching food in an area. There is only one piece of food in the area being searched. All the birds do not know where the food is. But they know how far the food is in their search (Cheng and Jin 2014). So the best strategy to attain the food is to simply follow the bird, which is nearest to the food. In optimization problems, each bird in the search space is referred to as 'particle'. All the particles are evaluated by the fitness function to be optimized and have velocities for 
Fig. 9 interaction of $a_{p}$ and $f_{z}$ plot for SR

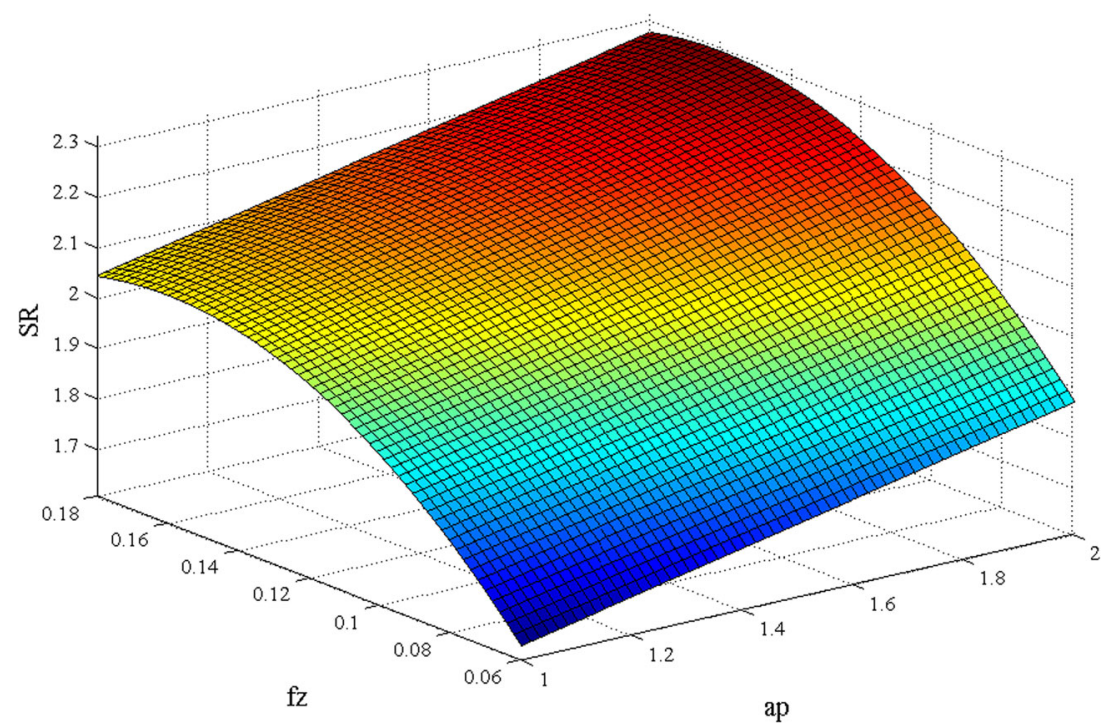

Table 6 Result of ANOVA for SR

\begin{tabular}{lllll}
\hline Machining parameters & Degree of freedom (Dof) & Sum of square $\left(\mathrm{SS}_{j}\right)$ & $P$ value & Contribution percentage $(\%)$ \\
\hline$V$ & 2 & 0.151400 & 0.005 & 24 \\
$f_{z}$ & 2 & 0.180067 & 0.012 & 29 \\
$v \times v$ & 2 & 0.000000 & 0.019 & 1 \\
$f_{z} \times f_{z}$ & 2 & 0.000000 & 0.035 & 1 \\
$V \times a_{p}$ & 4 & 0.257733 & 0.039 & 41 \\
Error & 3 & 0.000000 & 0.004 & - \\
Total & 15 & 0.58920 & - & - \\
\hline
\end{tabular}

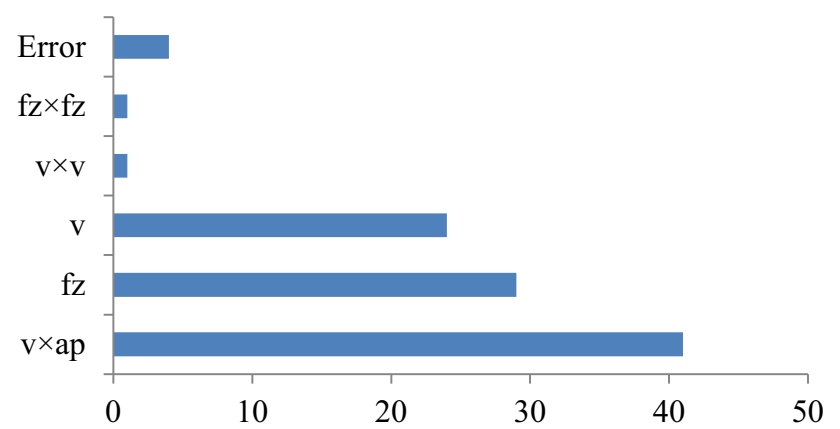

Fig. 10 The effect of machining parameters on the SR

the particles. The particles fly through the problem space by following the current optimum particles. The problem is initialized with a group of random particles and then searches for optima by updating generations. In all the iterations, each particle is updated by following two 'best' values. The best solution achieved so far among the particle is called as 'particle best' termed as pest and the best solution obtained so far in the population are called as 'global best' termed as g gest. A particle takes the entire particle toward its $\mathrm{p}_{\text {best }}$ and $\mathrm{g}_{\text {best }}$ locations (Bharathi Raja and Baskar 2012). After finding the two best values, the particles are updated with its velocity and positions using Eqs. (9) and (10) (Bharathi Raja and Baskar 2012)

$$
\begin{aligned}
V[]= & C_{1} \operatorname{rand}()(\operatorname{pbest}[]-\operatorname{present}[])+C_{2} \operatorname{rand}()(\operatorname{gbest}[] \\
& -\operatorname{present}[])
\end{aligned}
$$$$
p[]=V[]+\operatorname{present}[] .
$$

$V[]$ is the particle velocity, present is the current particle, $\mathrm{p}_{\text {best }}$ and $\mathrm{g}_{\text {best }}$ are defined as stated before, rand () is the random number between 0 and $1, c_{1}, c_{2}$ are learning factors usually varies from 1 to $4, p[]$ is new particle position. Compared to other optimization techniques, the information sharing mechanism in PSO is significantly different. Only $\mathrm{g}_{\text {best }}$ gives out information to others, which is a oneway information sharing mechanism. The evolution looks only for the best solution and hence all the particles tend to converge to the best solution quickly in most cases. The advantages of using PSO are that it takes real numbers as particles and there are few parameters to adjust.

The searching is a repeat process and the stop criteria are that the maximum iteration is reached or the minimum 
error condition is satisfied. The various parameters in PSO are number of particles, dimension of particles, and range of particles, learning factor, stop condition and global versus local version

\section{Algorithm}

Step 1 Initialize a population of $n$ particles randomly.

Step 2 Calculate fitness value for each particle. If the fitness value is better than the best fitness value ( $p_{\text {best }}$ ) in history, set current value as the new pest $_{\text {b }}$.

Step 3 Choose particle with the best fitness value of all the particles considered so far as the $\mathrm{g}_{\text {best }}$.

Step 4 For each particle; calculate particle velocity and position according to Eqs. (9) and (10).

Step 5 Particle velocities on each dimension are clamped to a maximum velocity $v_{\max }$. If the sum of acceleration would cause the velocity on that dimension to exceed $v_{\max }$ (Specified by the user), the velocity on the dimension is limited to $v_{\text {max }}$.

Step 6 Terminate if maximum number of iterations is reached.

Else, go to Step 2.

Step 7 End.

\section{Parameters of PSO}

The parameters of PSO technique used in the proposed mathematical model are given below.

Number of iteration performed: 30

Population: 50

Learning factor $c_{1}: 2$

Learning factor $c_{2}: 2$

\section{Calculation of optimum machining parameters}

Cutting speed is calculated randomly within the limits using Eq. (11) (Bharathi Raja and Baskar 2012)

$v=v_{\min }+\left(v_{\max }-v_{\min }\right) \operatorname{rand}()$

Similarly feed is also calculated randomly within the limits using Eq. (12).

$f z=f z_{\min }+\left(f z_{\max }-f z_{\min }\right) \operatorname{rand}()$

Similarly depth of cut is also calculated randomly within the limits using Eq. (13).

$\mathrm{ap}=\mathrm{ap}_{\min }+\left(\mathrm{ap}_{\max }-\mathrm{ap}_{\min }\right) \mathrm{rand}()$

\section{Confirmation of the proposed algorithm using simulated annealing algorithm}

Simulated annealing (SA) algorithm is an optimization process whose operation is strongly reminiscent of the physical annealing of crystalline compounds such as metals and metallic alloys (Kirkpatrick et al. 1983). In condensed matter physics, annealing is a physical process that is used to reconstruct the crystal structure of a solid with a low energy state. A solid in a state bath is first heated up to a temperature above the melting point of the solid. At this temperature, all particles of the solid are in violent random motion. The temperature of the heat bath is then slowly cooled down. All particles of the solid rearrange themselves and tend toward a low energy state. As the cooling of the particle is carried out sufficiently slowly, lower and lower energy states are obtained until the lowest energy state is reached. Similarly, in face milling an energy function is created which is minimized. While minimizing efforts are made to avoid local minima and to achieve global minima. The lowest energy level gives the optimized value of face milling parameters.

A standard SA procedure begins by generating an initial solution at random. At initial stages, a small random change is made in the current solution. Then the objective function value of new solution is calculated and compared with that of current solution. A move is made to the new solution if it has better value or if the probability function implemented in SA has a higher value than a randomly generated number. The probability of accepting a new solution is given as follows:

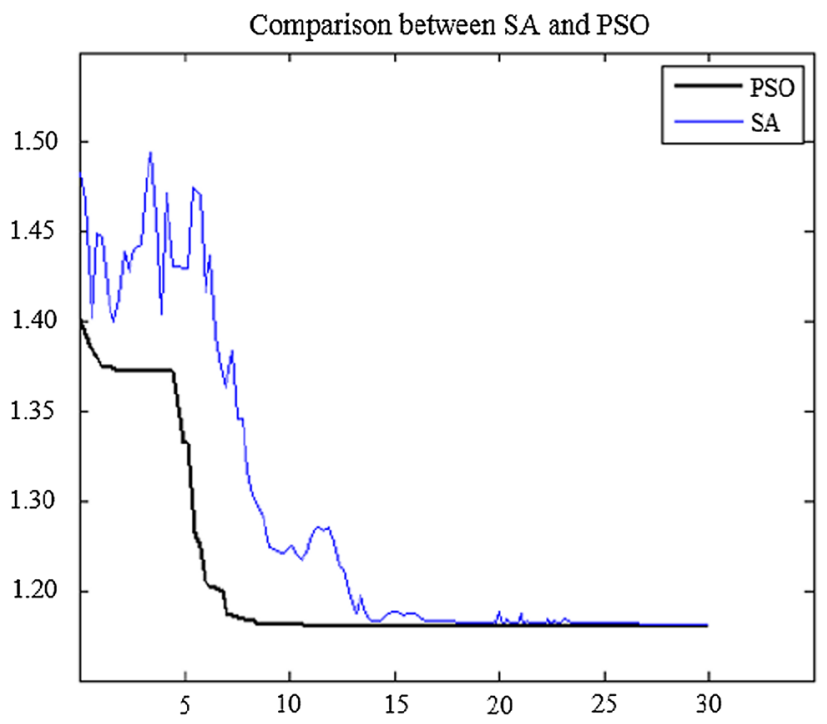

Fig. 11 PSO and SA convergence curve for SR 
Table 7 Experimental results of surface roughness for the optimized machining parameters

\begin{tabular}{lllllll}
\hline Machining characteristic & $V(\mathrm{opt})(\mathrm{m} / \mathrm{min})$ & $f_{z}(\mathrm{opt})(\mathrm{mm} /(\mathrm{rev} \times$ tooth $)$ & $a_{p}(\mathrm{opt})(\mathrm{mm})$ & Predicated value & Experimental value & Error $(\%)$ \\
\hline SR & 280 & 0.06 & 1 & 1.18 & 1.21 & 2.5 \\
\hline
\end{tabular}

$p=\left\{\begin{array}{cll}1 & \text { if } \quad \Delta<0 \\ e^{-\Delta / t} & \text { if } \quad \Delta \geq 0\end{array}\right.$

The calculation of this probability relies on a temperature parameter, $T$, which is referred to as temperature, since it plays a similar role as the temperature in the physical annealing process. To avoid getting trapped at a local minimum point, the rate of reduction should be slow (Kirkpatrick et al. 1983). In our problem the following method to reduce the temperature has been used:

$T_{i+1}=c T_{i} \quad i=0,1, \ldots \quad$ and $\quad 0.9 \leq c<1$

Thus, at the start of SA most worsening moves may be accepted, but at the end only improving ones are likely to be allowed. This can help the procedure jump out of a local minimum. The algorithm may be terminated after a certain volume fraction for the structure has been reached or after a pre-specified run time.

Simulated annealing algorithm has diverse applications including improving the performance of other artificial intelligence techniques and determining the optimal set of process parameter (Kirkpatrick et al. 1983; Yang et al. 2009). In this research, SA has been used in order to check the performance of the PSO. Results indicate that the proposed optimization procedure is quite efficient in optimization of face milling process parameters.

Optimization based on PSO and SA was executed using MATLAB software in which 30 iterations with 50 populations were used to run the program. The program is executed to get optimized machining parameters for minimizing SR. Figure 11 shows the convergence curve towards the optimal solution for SR.

\section{Validation of machining parameters}

The optimum machining parameters found using PSO is validated by conducting experiments on the same specification of AISI1045 material. Table 7 shows the results of the confirmation experiments.

\section{Results and discussion}

The effect of machining parameters on the surface roughness was considered. 9 experiments were conducted on AISI1045 and their corresponding surface roughness values measured. Then optimization based on PSO was executed using MATLAB software in which 30 iterations with
50 populations were used to run the program. The computational time for execution of single run in a Core 2 Duo processor computer is observed to be $15 \mathrm{~s}$ in an average. Then once again experiments were conducted based on the recommended machining parameters of PSO. It is observed from the conducted experiments, the surface roughness decreases with an increase in cutting speed and surface roughness decreases with a decrease in feed. The predicted surface roughness largely agrees with the experimental results. The difference $(2.5 \%)$ between the results of proposed technique and the experiments may be attributed to the effects of vibration, spindle run-out, and work piece material property in actual machining.

\section{Scope for future work}

In this work, the minimum surface roughness has been predicted using PSO and the same is confirmed by conducting experiments using the same parameters. But, machining is a complex phenomenon and so inclusion of many other machining parameters and constraints may enhance the end result. Cutting force, tool life and many other performance measures can be predicted using the proposed methodology. Multi-pass milling or component based attempts can be carried out to show the ability and effectiveness of non-traditional optimization techniques.

Open Access This article is distributed under the terms of the Creative Commons Attribution 4.0 International License (http://crea tivecommons.org/licenses/by/4.0/), which permits unrestricted use, distribution, and reproduction in any medium, provided you give appropriate credit to the original author(s) and the source, provide a link to the Creative Commons license, and indicate if changes were made.

\section{References}

Arrazola PJ, Özel T, Umbrello D, Davies M, Jawahir IS (2013) Recent advances in modeling of metal machining processes. CIRP Ann Manuf Technol 62:695-718

Asokan P, Kumar RR, Jeyapaul R, Santhi M (2008) Development of multi-objective optimization models for electrochemical machining process. Int J Mater Manuf Technol 39:55-63

Azadi Moghaddam M, Kolahan F (2014) Modeling and optimization of surface roughness of AISI2312 hot worked steel in EDM based on mathematical modeling and genetic algorithm. Int $\mathbf{J}$ Eng 27(3):417-424

Baji D, Lela B, Zivkovic D (2008) Modeling of machined surface roughness and optimization of cutting parameters in face milling. Metallurgical 47:331-334 
Bharathi Raja S, Baskar N (2012a) Application of particle swarm optimization technique for achieving desired milled surface roughness in minimum machining time. Expert Syst Appl 39:5982-5989

Bharathi Raja S, Baskar N (2012b) Application of particle swarm optimization technique for achieving desired milled surface roughness in minimum machining time. Expert Syst Appl 39:5982

Cheng R, Jin Y (2014) A social learning particle swarm optimization algorithm for scalable optimization. Inform Sci. doi:10.1016/j. ins.2014.08.039

Jeang A (2011) Cutting parameters optimization for prediction time via computer experiments. Appl Math Model 35:1354-1362

Jesuthanam C, Kumanan S, Asojan P (2007) Surface roughness prediction hybrid neural network. Mach Sci Technol 111:271-286

Kirkpatrick S, Gelatt J, Vecchi DD (1983) Optimization by simulated annealing. Science 220:671-680

Kolahan F, Bironro M (2008) Modeling and optimization of process parameters in PMEDM by genetic algorithm. WASET 36:480-484

Kolahan F, Golmezerji R, Azadi Moghaddam M (2012) Multi objective optimization of turning process using grey relational analysis and simulated annealing algorithm. Appl Mech Mater 110:2926-2932
Patricia Mũnoz-Escalona P, Maropoulos PG (2014) A geometrical model for surface roughness prediction when face milling $\mathrm{Al}$ 7075-T7351 with square insert tools. J Manuf Syst. doi:10.1016/ j.jmsy.2014.06.011

Reddy S, Venkateswara R (2006) Selection of optimum tool geometry and cutting conditions using a surface roughness prediction model for end milling. Int J Adv Manuf Technol 26:1202-1210

Roy RK (2010) A primer on the Taguchi method, 2nd edn. Society of Manufacturing Engineers, USA

Vishnu D, Asal RI, Patel T, Alok B (2013) Optimization of process parameters of EDM using ANOVA method. Int J Eng Res Appl 3(2):1119-1125

Vishwakarma M, Vishal Parashar VK (2012) Regression analysis and optimization of material removal rate on electric discharge machine for EN-19 alloy steel. Int J Sci Res Publ 2(11):167-175

Yang SH, Srinivas J, Mohan S, Lee DM, Balaji S (2009) Optimization of electric discharge machining using simulated annealing. J Mater Process Technol 209(9):4471-4475

Zhang J, Chenb J, Kirby D (2007) Surface roughness optimization in an end milling operation using the Taguchi design method. J Mater Process Technol 184:233 